\title{
Spatiotemporal gene expression profiles associated with male strobilus development in Cryptomeria japonica by suppression subtractive hybridization
}

\author{
Manabu Kurita ${ }^{1,2)}$, Toru Taniguchi1 ${ }^{1,2)}$, Ryogo Nakada $^{2)}$, Teiji Kondo ${ }^{2)}$ and Atsushi Watanabe*2) \\ 1) Forest Bio-Research Center, Forestry and Forest Products Research Institute, 3809-1 Ishi, Juo, Hitachi, Ibaraki 319-1301, Japan \\ 2) Forest Tree Breeding Center, Forestry and Forest Products Research Institute, 3809-1 Ishi, Juo, Hitachi, Ibaraki 319-1301, Japan
}

\begin{abstract}
To identify the genes associated with the formation and development of the male strobilus of Cryptomeria japonica D. Don (Sugi), male strobilus-specific suppression subtractive hybridization (SSH) libraries were constructed from three different stages of male strobilus development. From these SSH libraries, 1,012 unigene sets including 314 contigs were assembled from 2,448 sequences. The profiles of the genes isolated from the SSH libraries differed strongly according to developmental stage: $49 \%, 13 \%$ and $29 \%$ of genes were unique to the early stage (stage E), tetrad stage (stage T), and mature stage (stage M) SSH libraries, respectively. To evaluate the reliability of the SSH libraries, we focused on the eight genes strongly concentrated in the three SSH libraries and performed RT-PCR analysis. All genes tested were expressed more strongly in the male strobilus than in the shoot. Our findings suggest that the gene profiles associated with male strobilus development differ in both number and kind of dominantly expressed genes.
\end{abstract}

Key Words: Cryptomeria japonica, male strobilus, gene profiling, suppression subtractive hybridization.

\section{Introduction}

Cryptomeria japonica D. Don (Sugi) is one of the most important commercial coniferous tree species in Japan. However, $C$. japonica pollen-induced pollinosis is the most widespread allergic disease in Japan, affecting over $15 \%$ of the population. Rapid improvement of this public health issue using a conventional breeding approach is not possible, because successful breeding of coniferous tree requires a very long time. Genetic modification might be a powerful tool to shorten the time needed to breed trees compared with traditional breeding methods because it is able to induce the favorable traits by introduction of specific genes in trees without unnecessary genetic transitions. A method for Agrobacterium tumefaciens-mediated transformation of embryogenic tissue was recently developed for C. japonica (Taniguchi et al. 2008). Introduction of a male sterile trait by genetic modification of $C$. japonica is a viable approach to address the medical and public health problems of $C$. japonica polleninduced pollinosis. To use this approach for male sterilization, it is necessary to understand the molecular mechanism of the male strobilus and pollen formation of $C$. japonica.

Flower formation genes of $C$. japonica, such as the Class B MADS-box gene (Fukui et al. 2001) and functional analysis of FLORICAULA/LEAFY homolog genes (Shiokawa et al. 2008), have been studied. Global analyses of the expressed genes in mature pollen and the male strobilus of

Communicated by Darshan S. Brar

Received January 25, 2011. Accepted March 27, 2011.

*Corresponding author (e-mail: nabeatsu@affrc.go.jp)
C. japonica have been reported by Futamura et al. (2006, 2008). However, no profiling data are available of gene expression that is associated with different developmental stages of the male strobilus or pollen. We surmised that such spatiotemporal expression profiling would help us to understand male strobilus formation of $C$. japonica at the molecular level. Therefore, we created male strobilus-specific libraries associated with male strobilus development by a suppression subtractive hybridization ( $\mathrm{SSH}$ ) method.

SSH is an effective method of isolating genes that are expressed differentially between two tissues (Shukla et al. 2006, Sternberg et al. 2007). Using molecular hybridization and subtraction techniques, the SSH cDNA library approach reduces the cloning of abundantly expressed housekeeping genes or genes commonly expressed in both control and treated tissues, and thereby the SSH library comprises target tissue-specific genes. To isolate genes related to male strobilus formation and development, male strobilus-specific SSH libraries were derived from three different stages of male strobilus development. Herein, we describe the isolation of cDNAs that are expressed differentially in three different stages of $C$. japonica male strobilus development.

\section{Materials and Methods}

\section{Plant materials}

Male strobili and vegetative shoots were collected from adult $C$. japonica trees of Ohtawara 1, a plus tree clone of C. japonica, planted at a clone bank garden maintained at the Forest Tree Breeding Center (FTBC), Forestry and Forest Products Research Institute (FFPRI), Hitachi, Ibaraki, 
Japan. Gibberellic acid $\left(\mathrm{GA}_{3}\right)(100 \mathrm{ppm})$ was sprayed on the trees on 7 July 2005 to induce flowering. Male strobili were sampled beginning on 18 August. Roots were also collected from cuttings of Ohtawara 1 grown in a tissue culture bottle at $25^{\circ} \mathrm{C}$ under a $16-\mathrm{h}$ light/8-h dark cycle.

\section{Scanning electron microscopy (SEM)}

Male strobili were sampled to observe the stages of strobilus development by SEM. Strobili collected on 19 September (Sep), 20 Sep, 22 Sep, 25 Sep, 29 Sep, 2 October (Oct), 5 Oct, 9 Oct, 12 Oct, 17 Oct, 20 Oct, 26 Oct and 24 November (Nov) were dehydrated in a graduated series of ethanol to prevent dehydration-induced tissue shrinkage. Air-dried strobili were then cut along the axis using sharp razor blades to obtain smooth surfaces for SEM observation. The specimens were placed on brass specimen stubs with doublecarbon-coated tape, and were coated with platinum by a magnetron-sputtering device (JUC-5000, JEOL, Tokyo, Japan). The specimens were observed with an SEM (JSM$5800 \mathrm{LV}$, JEOL, Tokyo, Japan) with a $15-\mathrm{kV}$ accelerating voltage. Based on our SEM observations of the male strobilus, we selected three different developmental stages of strobilus samples collected on 19 Sep, 9 Oct and 24 Nov. These strobilus pollen development stages were named the early stage (stage E), the tetrad stage (stage T), and the mature stage (stage M), respectively (Fig. 1).

\section{Isolation of RNA and reverse transcription}

Male strobili were sampled simultaneously for RNA extraction. Total RNA was isolated from the tissue of male strobili (stage E, stage T, stage M) and shoots (17 May, 19 Sep, 9 Oct, 24 Nov) of mature trees and roots of cuttings using the RNeasy Plant Mini kit (QIAGEN, Gaithersburg, MD) with minor modifications. The cDNA synthesis was performed using the Super SMART PCR cDNA Synthesis kit (BD Biosciences, Franklin Lakes, NJ) according to the manufacturer's instructions. Single-strand cDNAs were produced by reverse transcription from $1 \mu \mathrm{g}$ total RNA of each sample.

\section{SSH}

We constructed three different subtraction libraries. The PCR-Select cDNA Subtraction kit (BD Biosciences) was used for the SSH to identify male strobilus-specific transcripts in C. japonica in a strategically designed experiment in which the male strobilus (stage $\mathrm{E}$, stage $\mathrm{T}$, stage $\mathrm{M}$, respectively) and shoot (19 Sep, 9 Oct, 24 Nov, respectively) cDNA populations served as 'tester' and 'driver', respectively. Double-stranded cDNA was synthesized and digested with restriction enzyme RsaI. Male strobilus cDNAs were divided into two groups and ligated to the specific adaptors 1 and 2R, respectively. Each denatured adaptor-ligated tester mix was separately hybridized with a single-stranded shoot cDNA driver for $8 \mathrm{~h}$ at $68^{\circ} \mathrm{C}$, to enrich nonpairing sequences unique to the male strobilus cDNA pool. A second hybridization using single-strand driver cDNA as well as the prod-


Fig. 1. Micrographs showing male flower development. Parts A, D and $\mathrm{G}$ are by optical microscope. The other parts are by SEM. A-C, specimens were taken at stage E (19 Sep); D-F, J, stage T (9 Oct; G-I, $\mathrm{K}$, stage $\mathrm{M}$ (24 Nov). J and $\mathrm{K}$ are enlargements of parts of $\mathrm{F}$ and I, respectively. Scale bars: $2 \mathrm{~mm}$ for A, D and G; $500 \mu \mathrm{m}$ for B, E and H; $100 \mu \mathrm{m}$ for $\mathrm{C}, \mathrm{F}$ and $\mathrm{I} ; 10 \mu \mathrm{m}$ for $\mathrm{J}$ and $\mathrm{K}$.

ucts of the first hybridization from both tester aliquots (adaptor 1- and adaptor 2R-linked molecules) was performed for $16 \mathrm{~h}$ at $68^{\circ} \mathrm{C}$. Products from the second hybridization were diluted in $200 \mu \mathrm{l}$ of dilution buffer of this kit, heated at $68^{\circ} \mathrm{C}$ for $7 \mathrm{~min}$, and stored at $-20^{\circ} \mathrm{C}$.

\section{PCR amplification of subtracted products}

An ABI 9700 Thermal Cycler (Applied Biosystems, Foster City, CA) was used to perform PCR amplification of the subtracted tester products. Primary PCR amplifications were conducted for each tester using diluted subtracted products following the second hybridization. A 1- $\mu$ l aliquot of sample was added to $24 \mu \mathrm{l}$ of PCR master mix prepared using the reagents supplied in the kit, and cycling conditions were as follows: $75^{\circ} \mathrm{C}$ for $5 \mathrm{~min}$ to extend the adaptors; $94^{\circ} \mathrm{C}$ for $25 \mathrm{~s}$; and 27 cycles at $94^{\circ} \mathrm{C}$ for $10 \mathrm{~s}, 66^{\circ} \mathrm{C}$ for $30 \mathrm{~s}$ and $72^{\circ} \mathrm{C}$ for $1.5 \mathrm{~min}$. Amplified products were diluted 100 -fold in sterile water, and $1 \mu \mathrm{l}$ of diluted primary PCR products was added to $24 \mu \mathrm{l}$ of secondary PCR master mix containing nested primers ( 1 and $2 \mathrm{R}$ ) to ensure the specific amplification of double-stranded templates containing both adaptors. Secondary PCR was performed at $94^{\circ} \mathrm{C}$ for $15 \mathrm{~s}$ and $68^{\circ} \mathrm{C}$ for $3 \mathrm{~min}$ (cycle numbers were 15, 18 and 15 for stage $\mathrm{E}$, stage $\mathrm{T}$ and stage $\mathrm{M}$, respectively). PCR products were analyzed on $2 \%$ agarose gel.

\section{Cloning of subtracted cDNA templates}

Following secondary PCR amplification, subtracted products from each tester cDNA population were cloned into pCR 4-TOPO using a TA Cloning kit (Invitrogen, Carlsbad, CA) and transformed into competent DH5 $\alpha$ Escherichia coli cells. Colonies were grown in a selective 
Table 1. Primers used for the RT-PCR

\begin{tabular}{|c|c|c|}
\hline Gene name & Forward primer & Reverse primer \\
\hline Cj_ssh_male_e_002 & 5'-CTGTTAAGTATCTTGTGCTTCACCAC-3' & 5'-CAACCACTGCCCCAACAATAGG-3' \\
\hline Cj_ssh_male_e_003 & 5'-AAAGCAGAATGGTGGTGCAGTG-3' & 5'-ATGCTCCAGACAAAACCGTAGG-3' \\
\hline Cj_ssh_male_t_001 & 5'-GCTGCACTGCCCTCAAATCG-3' & 5'-CCTTGAGAGCTACTTTATAGCATAG-3' \\
\hline Cj_ssh_male_t_002 & 5'-TGGCACAGGAAAAATCAGTCCAC-3' & 5'-TCGCCCATCAAACTATATGCATGATC-3' \\
\hline Cj_ssh_male_t_003 & 5'-CATAACATTGCATTCATTGCCTTGCAC-3' & 5'-ACCCTCAAGAACATTGAGCAGG-3' \\
\hline Cj_ssh_male_m_001 & 5'-CTGCCTGGTGTTGTTATTGTTGC-3' & 5'-GAAGACACAACACAAGTTGGTGAG-3' \\
\hline Cj_ssh_male_m_002 & 5'-ACAGACTGCCACAGATATACTTCTG-3' & 5'-ACAGATTGGCTGCCTTATTTTAGGC-3' \\
\hline Cj_ssh_male_m_003 & 5'-TTCGAGAAGTGCAAACTTGAGCG-3' & 5'-AGTCACTTGAGAATCAGCAGAGC-3' \\
\hline Actin & 5'-ATGTTTGAGACCTTCAATACACCA-3' & 5'-GAAGCTCATAACTCTTCTCAAGG-3' \\
\hline
\end{tabular}

Luria-Bertani agar medium containing X-gal and isopropyl $\beta$-D-1-thiogalactopyranoside for screening of blue/white colonies. For each tester, white colonies were randomly picked, placed on microtiter plates, and grown overnight in liquid Luria-Bertani medium.

\section{DNA sequencing and analysis}

$\mathrm{SSH}$ clone inserts were sequenced using a cloning vector primer (M13R) and an Applied Biosystems 3100 sequencer with the BigDye Terminator v3.1 cycle Sequencing kit (Applied Biosystems). The sequences were edited using Sequencher Software (Gene Codes Corporation, Ann Arbor, MI). Sequence analysis was performed using the BLASTX programs of the National Center for Biotechnology Information (NCBI) and The Arabidopsis Information Resource (TAIR).

\section{$R T-P C R$}

RT-PCR amplifications were performed with $1 \mu \mathrm{g}$ total RNA from the male strobilus (stage E, stage T, stage M), shoots (17 May, 19 Sep, 9 Oct, 24 Nov), and roots. Firststrand cDNA synthesis was performed using Superscript and oligo-dT primers (Invitrogen). RT-PCR primer sets are listed in Table 1. The reaction mixture for RT-PCR contained $0.5 \mu \mathrm{l}$ of the solution of the first-strand cDNA added to $49.5 \mu \mathrm{l}$ of KOD plus Master Mix (TOYOBO, Osaka, Japan) and $0.4 \mu \mathrm{M}$ of gene-specific primers (Table 1), for a total volume of $50 \mu \mathrm{l}$. The program used for RT-PCR was $94^{\circ} \mathrm{C}$ for $5 \mathrm{~min}$ followed by 25 cycles at $94^{\circ} \mathrm{C}$ for $30 \mathrm{~s}, 60^{\circ} \mathrm{C}$ for $30 \mathrm{~s}, 72^{\circ} \mathrm{C}$ for $30 \mathrm{~s}$ and finally $72^{\circ} \mathrm{C}$ for $1 \mathrm{~min}$. The PCR product was separated on $2 \%$ agarose gel and stained with SYBR SAFE (Invitrogen).

\section{Results}

Gene expression profile in the three developmental stages of male strobilus

A total of 2,448 clones was isolated and sequenced. The results of sequence analysis by BLASTX were filtered using an E-value of 1e-4 (Table 2; full data are shown in Supplemental Table 1). As a result of the BLASTX search, 458 unigene sets including 123 contigs were derived from 873 ESTs isolated from stage $\mathrm{E}$. The most frequently isolated genes in this stage had similarity with a bark protein-like protein and a pollen Ole e 1 allergen and extensin family protein (Table 2). Similarly, 727 ESTs isolated from the stage T library formed 175 unigene sets including 68 contigs. In the stage $\mathrm{T}$ library, there was a strong concentration of three genes. The most frequently isolated gene had similarity with Male cone protein 1 (PrMC1), the second most frequently isolated gene was a lipase, and the third was a PrMALE1 (Table 2). In stage M, 848 clones consisting of 379 unigene sets including 123 contigs were isolated. The most frequently isolated genes obtained from this stage were similar to SF18 from sunflower, CjMP1 of C. japonica, and peroxidase, respectively (Table 2). The profiles of the genes isolated from the SSH libraries differed strongly depending on the developmental stage of the strobilus: $49 \%, 13 \%$ and $29 \%$ of genes were isolated only from stage $\mathrm{E}, \mathrm{T}$ and $\mathrm{M} \mathrm{SSH}$ libraries, respectively (Fig. 2). The genes isolated from $\mathrm{SSH}$ libraries of all stages constituted only $1 \%$ of all genes (Fig. 2). For the purpose of the analysis of the trait of each developmental stage, each library-specific gene was analyzed by TAIR GO annotation search (Fig. 3) (http:// www.arabidopsis.org/index.jsp). In stage E, many genes were categorized into cell organization and biogenesis compared with other libraries; in stage T, many genes were categorized into metabolic processes and unknown biological processes compared with other libraries. Many genes categorized into response to stress compared with other libraries were included in stage M (Fig. 3).

\section{Expression analysis of the genes isolated from SSH libraries}

Although SSH can be used to isolate spatiotemporalspecific genes, the reliability of the $\mathrm{SSH}$ libraries must be evaluated. Therefore, we evaluated their reliability by RTPCR analysis. The eight genes that were main components of each SSH library were used for RT-PCR (from stage E: Cj_ssh_male_e_002 (3.4\%) and Cj_ssh_male_e_003 $(2.1 \%)$ : from stage $\mathrm{T}$ : Cj_ssh_male_t_001 $(24 \%)$, Cj_ssh_male_t_002 $(22 \%)$ and $\bar{C} j \_s s h$ male_t_003 (14\%): from stage M: Cj_ssh_male_m_o0 $\overline{1} \quad(12 \%)$, Cj_ssh_male_m_002 $(5.1 \%)$ and $C j \_s s h$ male_m_003 $(2.8 \%)$ (Table 2)).

We designed gene-specific primers for each gene (Table 1), and investigated the patterns of expression 
Table 2. The twenty most highly expressed unigenes in the stage E, T and M SSH libraries

\begin{tabular}{|c|c|c|c|c|c|}
\hline Gene ID & Accession No. & $\begin{array}{l}\text { \# ESTs (\% in } \\
\text { the library) }\end{array}$ & $\begin{array}{l}\text { highest homology (NCBI) } \\
\text { highest homology (TAIR) }\end{array}$ & $\begin{array}{l}\text { Homology accession } \\
\text { locus name }\end{array}$ & E-value \\
\hline \multicolumn{6}{|l|}{ Stage E } \\
\hline Cj_ssh_male_e_001 & FS942126 & $\begin{array}{c}30 \\
(3.4)\end{array}$ & $\begin{array}{l}\text { bark protein-like protein } \\
\text { catalytic }\end{array}$ & $\begin{array}{l}\text { AAV65286 } \\
\text { AT4G28940.1 }\end{array}$ & $\begin{array}{l}2 e-73 \\
1 e-59\end{array}$ \\
\hline Cj_ssh_male_e_002 & FS942127 & $\begin{array}{c}30 \\
(3.4)\end{array}$ & $\begin{array}{l}\text { unknown } \\
\text { pollen Ole e } 1 \text { allergen and extensin family protein }\end{array}$ & $\begin{array}{l}\text { ABK26139 } \\
\text { AT5G15780.1 }\end{array}$ & $\begin{array}{l}9 \mathrm{e}-18 \\
9 \mathrm{e}-10\end{array}$ \\
\hline Cj_ssh_male_e_003 & FS942128 & $\begin{array}{l}18 \\
(2.1)\end{array}$ & $\begin{array}{l}\text { unknown } \\
\text { unknown protein }\end{array}$ & $\begin{array}{l}\text { ABK22759 } \\
\text { AT5G48480.1 }\end{array}$ & $\begin{array}{l}9 e-63 \\
6 e-21\end{array}$ \\
\hline Cj_ssh_male_e_004 & FS942129 & $\begin{array}{c}17 \\
(1.9)\end{array}$ & $\begin{array}{l}\text { unknown } \\
\text { MT3 (METALLOTHIONEIN 3) }\end{array}$ & $\begin{array}{l}\text { ABK21034 } \\
\text { AT3G15353.1 }\end{array}$ & $\begin{array}{l}4 e-10 \\
1 e-04\end{array}$ \\
\hline Cj_ssh_male_e_005 & FS942130 & $\begin{array}{c}15 \\
(1.7)\end{array}$ & conserved hypothetical protein & YP_001680918 & $8 \mathrm{e}-28$ \\
\hline Cj_ssh_male_e_006 & FS942131 & $\begin{array}{c}14 \\
(1.6)\end{array}$ & hypothetical protein & CAN70790 & $9 e-36$ \\
\hline Cj_ssh_male_e_007 & FS942132 & $\begin{array}{c}13 \\
(1.5)\end{array}$ & hypothetical protein PhapfoPp090 & YP_358636 & $6 e-45$ \\
\hline Cj_ssh_male_e_008 & FS942133 & $\begin{array}{c}11 \\
(1.3)\end{array}$ & $\begin{array}{l}\text { putative cyclosporin A-binding protein } \\
\text { ROC1 (ROTAMASE CYP 1); peptidyl-prolyl cis- } \\
\text { trans isomerase }\end{array}$ & $\begin{array}{l}\text { CAC } 81066 \\
\text { AT4G38740.1 }\end{array}$ & $\begin{array}{l}1 e-56 \\
2 e-53\end{array}$ \\
\hline Cj_ssh_male_e_009 & FS942134 & $\begin{array}{c}11 \\
(1.3)\end{array}$ & $\begin{array}{l}\text { limonene/borneol synthase } \\
\text { terpene synthase/cyclase family protein }\end{array}$ & $\begin{array}{l}\text { BAC92722 } \\
\text { AT3G29410.1 }\end{array}$ & $\begin{array}{l}7 e-44 \\
2 e-17\end{array}$ \\
\hline Cj_ssh_male_e_010 & FS942135 & $\begin{array}{c}10 \\
(1.1)\end{array}$ & hypothetical protein & CAN65763 & $5 e-25$ \\
\hline Cj_ssh_male_e_011 & FS942136 & $\begin{array}{c}9 \\
(1.0)\end{array}$ & $\begin{array}{l}\text { translation elongation factor-1 alpha } \\
\text { elongation factor 1-alpha }\end{array}$ & $\begin{array}{l}\text { AAV92325 } \\
\text { AT5G60390.3 }\end{array}$ & $\begin{array}{l}2 e-123 \\
e-117\end{array}$ \\
\hline Cj_ssh_male_e_012 & FS942137 & $\begin{array}{c}8 \\
(0.9)\end{array}$ & $\begin{array}{l}\text { unknown } \\
\text { xyloglucan:xyloglucosyl transferase, putative }\end{array}$ & $\begin{array}{l}\text { ABK22211 } \\
\text { AT3G23730.1 }\end{array}$ & $\begin{array}{l}3 e-97 \\
1 e-77\end{array}$ \\
\hline Cj_ssh_male_e_013 & FS942138 & $\begin{array}{c}8 \\
(0.9)\end{array}$ & unnamed protein product & CAO18260 & $2 \mathrm{e}-24$ \\
\hline Cj_ssh_male_e_014 & FS942139 & $\begin{array}{c}8 \\
(0.9)\end{array}$ & $\begin{array}{l}\text { unknown } \\
\text { LTP; lipid binding }\end{array}$ & $\begin{array}{l}\text { ABK21262 } \\
\text { AT2G15050.3 }\end{array}$ & $\begin{array}{l}2 \mathrm{e}-18 \\
6 \mathrm{e}-08\end{array}$ \\
\hline Cj_ssh_male_e_015 & FS942140 & $\begin{array}{c}8 \\
(0.9)\end{array}$ & hypothetical protein Bm1_17870 & XP_001895031 & $4 \mathrm{e}-18$ \\
\hline Cj_ssh_male_e_016 & FS942141 & $\begin{array}{c}7 \\
(0.8)\end{array}$ & $\begin{array}{l}\text { unknown } \\
\text { pollen Ole e } 1 \text { allergen and extensin family protein }\end{array}$ & $\begin{array}{l}\text { ABK24101 } \\
\text { AT5G15780.1 }\end{array}$ & $\begin{array}{l}7 e-20 \\
1 e-11\end{array}$ \\
\hline Cj_ssh_male_e_017 & FS942142 & $\begin{array}{c}6 \\
(0.7)\end{array}$ & $\begin{array}{l}\text { Os } 01 \mathrm{~g} 0317800 \\
\text { esterase/lipase/thioesterase family protein }\end{array}$ & $\begin{array}{l}\text { NP_001042883 } \\
\text { AT5G19290.1 }\end{array}$ & $\begin{array}{l}4 e-62 \\
1 e-63\end{array}$ \\
\hline Cj_ssh_male_e_018 & FS942143 & $\begin{array}{c}5 \\
(0.6)\end{array}$ & $\begin{array}{l}\text { flavonol synthase } \\
\text { FLS (FLAVONOL SYNTHASE) }\end{array}$ & $\begin{array}{l}\text { AAS } 21058 \\
\text { AT5G08640.1 }\end{array}$ & $\begin{array}{l}1 e-80 \\
1 e-67\end{array}$ \\
\hline Cj_ssh_male_e_019 & FS942144 & $\begin{array}{c}5 \\
(0.6)\end{array}$ & $\begin{array}{l}\text { unknown } \\
\text { glycosyl hydrolase family } 17 \text { protein }\end{array}$ & $\begin{array}{l}\text { ABK92452 } \\
\text { AT2G05790.1 }\end{array}$ & $\begin{array}{l}2 e-62 \\
3 e-62\end{array}$ \\
\hline Cj_ssh_male_e_020 & FS942145 & $\begin{array}{c}5 \\
(0.6)\end{array}$ & $\begin{array}{l}\text { delta-selinene synthase } \\
\text { GA1 (GA REQUIRING 1); ent-copalyl diphosphate } \\
\text { synthase }\end{array}$ & $\begin{array}{l}\text { AAK83561 } \\
\text { AT4G02780.1 }\end{array}$ & $\begin{array}{l}9 e-41 \\
3 e-16\end{array}$ \\
\hline Stage $T$ & & & & & \\
\hline Cj_ssh_male_t_001 & FS942584 & $\begin{array}{l}175 \\
(24)\end{array}$ & $\begin{array}{l}\text { Male-cone protein } 1 \text { precursor (PRMC1) } \\
\text { protease inhibitor/seed storage/lipid transfer protein } \\
\text { (LTP) family protein }\end{array}$ & $\begin{array}{l}\text { O24493 } \\
\text { AT5G62080.1 }\end{array}$ & $\begin{array}{l}1 e-15 \\
1 e-13\end{array}$ \\
\hline Cj_ssh_male_t_002 & FS942585 & $\begin{array}{l}161 \\
(22)\end{array}$ & $\begin{array}{l}\text { similar to Bile-salt-activated lipase precursor (BAL) } \\
\text { protein kinase family protein }\end{array}$ & $\begin{array}{l}\text { XP_859406 } \\
\text { AT1G49270.1 }\end{array}$ & $\begin{array}{l}2 \mathrm{e}-08 \\
1 \mathrm{e}-05\end{array}$ \\
\hline Cj_ssh_male_t_003 & FS942586 & $\begin{array}{l}104 \\
(14)\end{array}$ & $\begin{array}{l}\text { PrMALE1 } \\
\text { protease inhibitor/seed storage/lipid transfer protein } \\
\text { (LTP) family protein }\end{array}$ & $\begin{array}{l}\text { AAD02170 } \\
\text { AT5G62080.1 }\end{array}$ & $\begin{array}{l}6 e-18 \\
4 e-08\end{array}$ \\
\hline Cj_ssh_male_t_004 & FS942587 & $\begin{array}{c}10 \\
(1.3)\end{array}$ & $\begin{array}{l}\text { unknown } \\
\text { MT3 (METALLOTHIONEIN 3) }\end{array}$ & $\begin{array}{l}\text { ABK21034 } \\
\text { AT3G15353.1 }\end{array}$ & $\begin{array}{l}1 e-10 \\
6 e-05\end{array}$ \\
\hline Cj_ssh_male_t_005 & FS942588 & $\begin{array}{c}6 \\
(0.8)\end{array}$ & $\begin{array}{l}\text { defensin } \\
\text { PDF2.1; peptidase inhibitor }\end{array}$ & $\begin{array}{l}\text { ABO61349 } \\
\text { AT2G02120.1 }\end{array}$ & $\begin{array}{l}3 e-23 \\
1 e-10\end{array}$ \\
\hline
\end{tabular}


Table 2. (continued)

\begin{tabular}{|c|c|c|c|c|c|}
\hline Gene ID & Accession No. & $\begin{array}{l}\text { \# ESTs (\% in } \\
\text { the library) }\end{array}$ & $\begin{array}{l}\text { highest homology (NCBI) } \\
\text { highest homology (TAIR) }\end{array}$ & $\begin{array}{l}\text { Homology accession } \\
\text { locus name }\end{array}$ & E-value \\
\hline \multirow[t]{2}{*}{$\overline{C j \_s s h \_m a l e \_t \_006}$} & FS942589 & 6 & predicted protein & XP_001774076 & $9 \mathrm{e}-30$ \\
\hline & & $(0.8)$ & kinesin light chain-related & AT3G27960.1 & $2 \mathrm{e}-30$ \\
\hline \multirow[t]{2}{*}{ Cj_ssh_male_t_007 } & FS942590 & 5 & unknown & ABK21262 & $2 \mathrm{e}-18$ \\
\hline & & $(0.7)$ & LTP; lipid binding & AT2G15050.3 & $6 e-08$ \\
\hline \multirow[t]{2}{*}{ Cj_ssh_male_t_008 } & FS942591 & 5 & unnamed protein product & CAO44892 & $5 e-50$ \\
\hline & & $(0.7)$ & LPD1 (LIPOAMIDE DEHYDROGENASE 1) & AT3G16950.2 & $5 e-51$ \\
\hline \multirow[t]{2}{*}{ Cj_ssh_male_t_009 } & FS942592 & 4 & unknown & ABK22309 & $1 \mathrm{e}-19$ \\
\hline & & $(0.6)$ & $\begin{array}{l}\text { protease inhibitor/seed storage/lipid transfer protein } \\
\text { (LTP) family protein }\end{array}$ & AT5G64080.2 & $7 \mathrm{e}-14$ \\
\hline \multirow[t]{2}{*}{ Cj_ssh_male_t_010 } & FS942593 & 4 & unknown & ABK23412 & $2 \mathrm{e}-12$ \\
\hline & & $(0.6)$ & AWPM-19-like membrane family protein & AT1G29520.1 & $6 e-08$ \\
\hline \multirow[t]{2}{*}{ Cj_ssh_male_t_011 } & FS942594 & 4 & unknown & ABK23967 & $2 \mathrm{e}-63$ \\
\hline & & $(0.6)$ & ADF6 (ACTIN DEPOLYMERIZING FACTOR 6) & AT2G31200.1 & $1 \mathrm{e}-55$ \\
\hline \multirow[t]{2}{*}{ Cj_ssh_male_t_012 } & FS942595 & 4 & Os01g0151700 & NP_001042042 & $7 e-93$ \\
\hline & & $(0.6)$ & $\begin{array}{l}\text { short-chain dehydrogenase/reductase (SDR) family } \\
\text { protein }\end{array}$ & AT5G 02540.1 & $1 \mathrm{e}-93$ \\
\hline \multirow[t]{2}{*}{ Cj_ssh_male_t_013 } & FS942596 & 4 & esterase/lipase/thioesterase family protein & NP_197430 & $4 e-62$ \\
\hline & & $(0.6)$ & esterase/lipase/thioesterase family protein & AT5̄G19290.1 & $5 e-64$ \\
\hline Cj_ssh_male_t_014 & FS942597 & $\begin{array}{c}3 \\
(0.4)\end{array}$ & Peptidase aspartic, active site & ABD33091 & $1 \mathrm{e}-08$ \\
\hline Cj_ssh_male_t_015 & FS942598 & $\begin{array}{c}3 \\
(0.4)\end{array}$ & unknown & ABK22573 & $3 e-15$ \\
\hline \multirow[t]{2}{*}{ Cj_ssh_male_t_016 } & FS942599 & 3 & unknown & ABK24327 & $5 e-59$ \\
\hline & & $(0.4)$ & calreticulin 2 (CRT2) & AT1G09210.1 & $1 e-58$ \\
\hline \multirow[t]{2}{*}{ Cj_ssh_male_t_017 } & FS942600 & 3 & male sterility-related protein & ACA28679 & $5 e-39$ \\
\hline & & $(0.4)$ & MS2 (MALE STERILITY 2) & AT3G11980.1 & $6 e-40$ \\
\hline \multirow[t]{2}{*}{ Cj_ssh_male_t_018 } & FS942601 & 3 & hypothetical protein & BAF00799 & $7 \mathrm{e}-08$ \\
\hline & & $(0.4)$ & $\begin{array}{l}\text { BEST A. thaliana protein match is: unknown protein } \\
\text { (TAIR:AT3G49590.2) }\end{array}$ & AT3G18770.1 & $3 e-11$ \\
\hline \multirow[t]{2}{*}{ Cj_ssh_male_t_019 } & FS942602 & 3 & hypothetical protein & CAN75831 & $8 e-42$ \\
\hline & & $(0.4)$ & GDSL-motif lipase/hydrolase family protein & AT2G23540.1 & $5 e-41$ \\
\hline \multirow[t]{2}{*}{ Cj_ssh_male_t_020 } & FS942603 & 3 & unnamed protein product & CAO46903 & $1 \mathrm{e}-32$ \\
\hline & & $(0.4)$ & ADL6 (DYNAMIN-LIKE PROTEIN 6); GTPase & AT1G10290.1 & $1 \mathrm{e}-30$ \\
\hline \multicolumn{6}{|l|}{ Stage M } \\
\hline \multirow{2}{*}{ Cj_ssh_male_m_001 } & FS942760 & 101 & Anther-specific protein SF18 & P22357 & $2 \mathrm{e}-05$ \\
\hline & & (12) & PDF1.3 (plant defensin 1.3) & AT2G26010.1 & $3 e-05$ \\
\hline Cj_ssh_male_m_002 & FS942761 & $\begin{array}{c}43 \\
(5.1)\end{array}$ & CjMP1 & BAE93119 & $1 \mathrm{e}-28$ \\
\hline \multirow[t]{2}{*}{ Cj_ssh_male_m_003 } & FS942762 & 24 & peroxidase & CAH 10840 & $1 \mathrm{e}-59$ \\
\hline & & $(2.8)$ & peroxidase, putative & AT5G05340.1 & $1 e-46$ \\
\hline \multirow[t]{2}{*}{ Cj_ssh_male_m_004 } & FS942763 & 20 & unknown & ABK 21034 & $3 e-10$ \\
\hline & & $(2.4)$ & MT3 (METALLOTHIONEIN 3) & AT3G15353.1 & $1 \mathrm{e}-04$ \\
\hline Cj_ssh_male_m_005 & FS942764 & $\begin{array}{c}19 \\
(2.2)\end{array}$ & hypothetical protein & CAN78649 & $5 \mathrm{e}-08$ \\
\hline \multirow[t]{2}{*}{ Cj_ssh_male_m_006 } & FS942765 & 16 & peroxidase & CAH10839 & $2 \mathrm{e}-48$ \\
\hline & & (1.9) & peroxidase, putative & AT5G05340.1 & $1 e-39$ \\
\hline Cj_ssh_male_m_007 & FS942766 & 12 & unknown & ACG33260 & $7 e-16$ \\
\hline & & (1.4) & unknown protein & AT5G55620.1 & $5 e-15$ \\
\hline Cj_ssh_male_m_008 & FS942767 & $\begin{array}{c}12 \\
(1.4)\end{array}$ & RNase $\mathrm{H}$ family protein & ABD63162 & $2 \mathrm{e}-34$ \\
\hline Cj_ssh_male_m_009 & FS942768 & 7 & unknown & ACG36115 & $5 e-16$ \\
\hline & & $(0.8)$ & unknown protein & AT5G55620.1 & $5 e-15$ \\
\hline Cj_ssh_male_m_010 & FS942769 & 7 & unknown & ABK20934 & $1 \mathrm{e}-11$ \\
\hline & & $(0.8)$ & MT2A (METALLOTHIONEIN 2A) & AT3G09390.1 & $2 \mathrm{e}-06$ \\
\hline Cj_ssh_male_m_011 & FS942770 & $\begin{array}{c}7 \\
(0.8)\end{array}$ & pollen allergen & BAF32143 & $2 \mathrm{e}-06$ \\
\hline
\end{tabular}


Table 2. (continued)

\begin{tabular}{|c|c|c|c|c|c|}
\hline Gene ID & Accession No. & $\begin{array}{l}\text { \# ESTs (\% in } \\
\text { the library) }\end{array}$ & $\begin{array}{l}\text { highest homology (NCBI) } \\
\text { highest homology (TAIR) }\end{array}$ & $\begin{array}{l}\text { Homology accession } \\
\text { locus name }\end{array}$ & E-value \\
\hline Cj_ssh_male_m_012 & FS942771 & $\begin{array}{c}7 \\
(0.8)\end{array}$ & Gag/pol polyprotein, 3'-partial, putative & ABI34371 & $2 \mathrm{e}-10$ \\
\hline Cj_ssh_male_m_013 & FS942772 & $\begin{array}{c}7 \\
(0.8)\end{array}$ & Peptidase aspartic, active site & ABD33091 & $2 \mathrm{e}-09$ \\
\hline Cj_ssh_male_m_014 & FS942773 & $\begin{array}{c}6 \\
(0.7)\end{array}$ & $\begin{array}{l}\text { translation elongation factor- } 1 \text { alpha } \\
\text { elongation factor } 1 \text {-alpha }\end{array}$ & $\begin{array}{l}\text { AAV92325 } \\
\text { AT5G60390.3 }\end{array}$ & $\begin{array}{l}2 e-123 \\
e-118\end{array}$ \\
\hline Cj_ssh_male_m_015 & FS942774 & $\begin{array}{c}6 \\
(0.7)\end{array}$ & $\begin{array}{l}\text { hypothetical protein } \\
\text { RNA binding / nucleic acid binding / nucleotide bind- } \\
\text { ing }\end{array}$ & $\begin{array}{l}\text { CAN70556 } \\
\text { AT5G66010.1 }\end{array}$ & $\begin{array}{l}4 e-72 \\
6 e-63\end{array}$ \\
\hline Cj_ssh_male_m_016 & FS942775 & $\begin{array}{c}6 \\
(0.7)\end{array}$ & unnamed protein product & BAB08213 & $1 e-37$ \\
\hline Cj_ssh_male_m_017 & FS942776 & $\begin{array}{c}5 \\
(0.6)\end{array}$ & $\begin{array}{l}\text { unknown } \\
\text { GPT2; antiporter/ glucose-6-phosphate transmem- } \\
\text { brane transporter }\end{array}$ & $\begin{array}{l}\text { ABK24716 } \\
\text { AT1G61800.1 }\end{array}$ & $\begin{array}{l}4 e-148 \\
e-130\end{array}$ \\
\hline Cj_ssh_male_m_018 & FS942777 & $\begin{array}{c}5 \\
(0.6)\end{array}$ & $\begin{array}{l}\text { unknown } \\
\text { AILP1 }\end{array}$ & $\begin{array}{l}\text { ABK21853 } \\
\text { AT5G19140.1 }\end{array}$ & $\begin{array}{l}9 e-93 \\
4 e-77\end{array}$ \\
\hline Cj_ssh_male_m_019 & FS942778 & $\begin{array}{c}5 \\
(0.6)\end{array}$ & $\begin{array}{l}\text { hypothetical protein OsJ_034246 } \\
\text { HSP93-III }\end{array}$ & $\begin{array}{l}\text { EAZ20037 } \\
\text { AT3G48870.1 }\end{array}$ & $\begin{array}{l}3 e-60 \\
4 e-61\end{array}$ \\
\hline Cj_ssh_male_m_020 & FS942779 & $\begin{array}{c}5 \\
(0.6)\end{array}$ & $\begin{array}{l}\text { ribosomal protein L18A } \\
60 \text { S ribosomal protein L18A (RPL18aB) }\end{array}$ & $\begin{array}{l}\text { AAT08714 } \\
\text { AT2G34480.1 }\end{array}$ & $\begin{array}{l}3 e-16 \\
9 e-18\end{array}$ \\
\hline
\end{tabular}

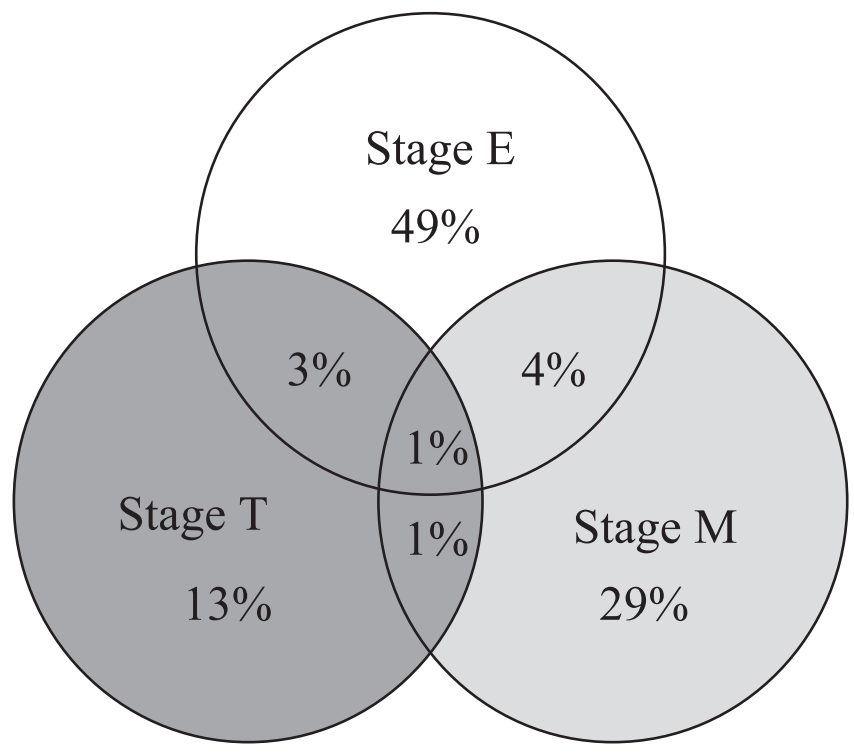

Fig. 2. Venn diagram showing the overlaps between SSH libraries. The genes that have sequence similarity with NCBI database (Evalue $<1 \mathrm{e}-4)$ were used. Stage E: early stage of pollen development, stage T: tetrad stage, stage M: mature pollen stage. The genes used for this analysis are shown in the Supplemental Table 1.

associated with the eight genes in each tissue of $C$. japonica using semi-quantitative RT-PCR (Fig. 4). In stage E, $C j \_s s h$ male_e_002 showed stronger expression in the male strobilus than in the shoot. Cj_ssh_male_e_003 showed the strongest expression in stage E male strobilus (Fig. 4). The other genes isolated from stage $\mathrm{T}$ and $\mathrm{M}$ SSH libraries were expressed only in male strobilus tissue. $C j \_s s h$ male_t_001,
$C j \_s s h \_m a l e \_t \_002$ and $C j \_s s h \_m a l e \_t \_003$ were strongly detected in stage $\mathrm{T}$ male strobilus tissue, and Cj_ssh_male_t_003 expression was also detected in stage $\mathrm{E}$ male strobilus tissue (Fig. 4). Similarly, Cj_ssh_male_m_001, Cj_ssh_male_m_002 and Cj_ssh_male_m_003 were detected only in stage $\mathrm{M}$ male strobilus tissue (Fig. 4). None of the eight genes were expressed in root tissue (Fig. 4).

\section{Discussion}

$\mathrm{SSH}$ is an effective method for isolating genes that are differentially expressed between two tissues (Shukla et al. 2006, Sternberg et al. 2007). We focused on isolating genes from the three different stages of male strobilus development, early, tetrad and mature pollen, of C. japonica (Fig. 1), using the SSH method. Ma et al. (2008) reported that approximately $80 \%$ of 15,950 transcripts shared across all anther stages are also expressed in leaves. Becker et al. (2003) analyzed transcriptional profiling of Arabidopsis pollen. Of the 1,584 genes detected in pollen, 1,422 (90\%) showed an overlap with the genes detected in vegetative tissues. These observations showed that there were very few tissue-specific transcripts. Although Futamura et al. (2008) reported the construction of a full-length cDNA library of the male strobilus of $C$. japonica and globally characterized expressed genes, many of the genes they isolated may not show male strobilus-specific expression. Moreover, the gene profiling associated with each developmental stage of the male strobilus of $C$. japonica has not been reported. We subtracted the housekeeping genes using the shoot as a driver, thereby effectively isolating male strobilus-specific genes. 


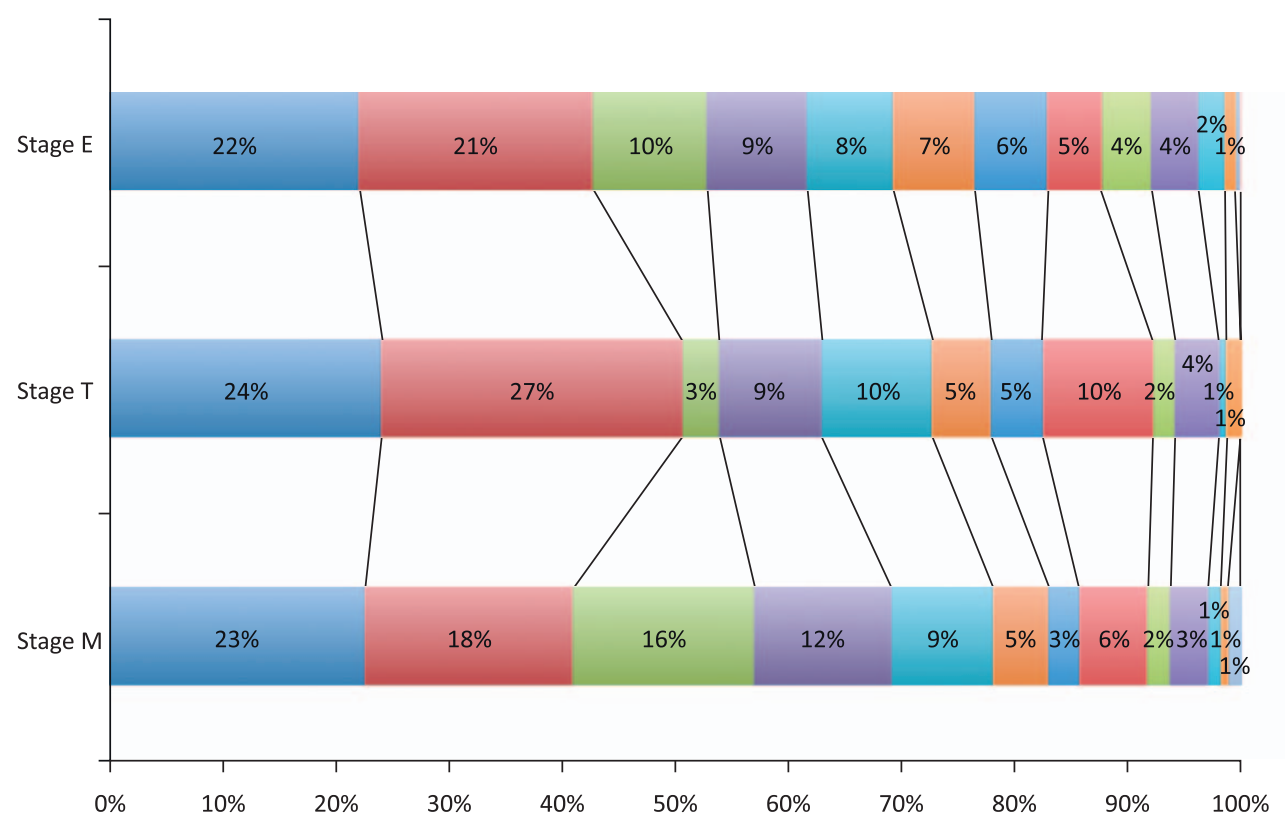

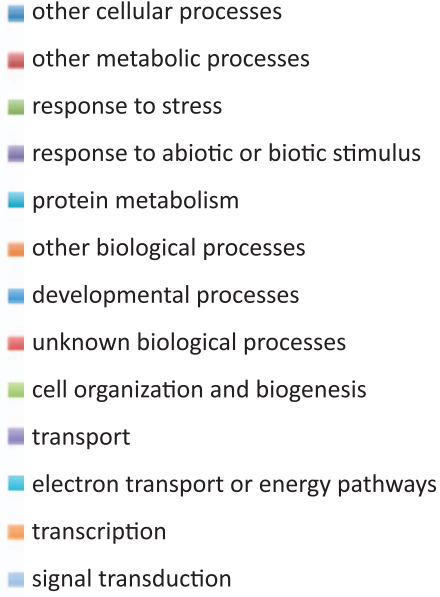

DNA or RNA metabolism

Fig. 3. Overview of the functional classification of stage-unique genes listed in Table 2 and Supplemental Table 1. Sequence homology search was analyzed by BLASTX program of TAIR (E-value $<1 \mathrm{e}-4$ ). The highest hits locus names were used for functional classification analysis by TAIR GO annotation search. Stage E: early stage of pollen development, stage T: tetrad stage, stage M: mature pollen stage.

We evaluated the SSH libraries by RT-PCR (Fig. 4), and believe that the subtraction was successful. Cj_ssh_male_e_002 and $C j \_s s h \_m a l e \_e \_003$ were also expressed in shoot. In C. japonica, male strobilus primordium is formed in the axil of the shoot (Fukui et al. 2001, Hashizume et al. 1973, Nagao et al. 1989). Stage $\mathrm{E}$ is an early stage of differentiation, so it has been assumed that the qualitative border of male reproductive tissue and vegetative tissue was not yet clear. Nevertheless, the genes that were expressed more strongly in the male strobilus than in shoot, such as $C j \_s s h$ male e 002 and $C j$ ssh male e 003, were isolated from our library. This result shows that SSH is an effective method for isolating genes that also differ quantitatively in expression. The other genes analyzed by RT-PCR showed not only tissue-specific expression but also stagespecific expression (Fig. 4).

In stage $\mathrm{E}$, the genes that have sequence similarity with the genes expressed in flower tissue were isolated (e.g., Cj_ssh_male_e_002, Cj_ssh_male_e_003 and Cj_ssh male e 010). The genes expressed in meristematic cells, which actively produced protein, were also isolated (e.g., Cj_ssh_male_e_011 and Cj_ssh_male_e_020). Cj_ssh male_e_002 and $C j$ ssh male e $e_{-} 01 \overline{6}$ had sequence similarity with Pollen Ole e 1 family protein (Table 2 ). Pollen Ole e 1 gene was expressed in pollen (Alche et al. 1999). Cj_ssh_male_e_003 is similar to early tobacco anther 1 (Accession No. AAO43000) (data not shown). Cj_ssh_male_e_011 and Cj_ssh_male e 020 had sequence similarity with translation elongation factor $(E F-l \alpha$ and $G A$ requiring 1 (GA1) genes, respectively. A high level of $E F-l \alpha$ expression was detected in the regions of high protein synthesis, including meristems, rapidly growing tissues, and de-

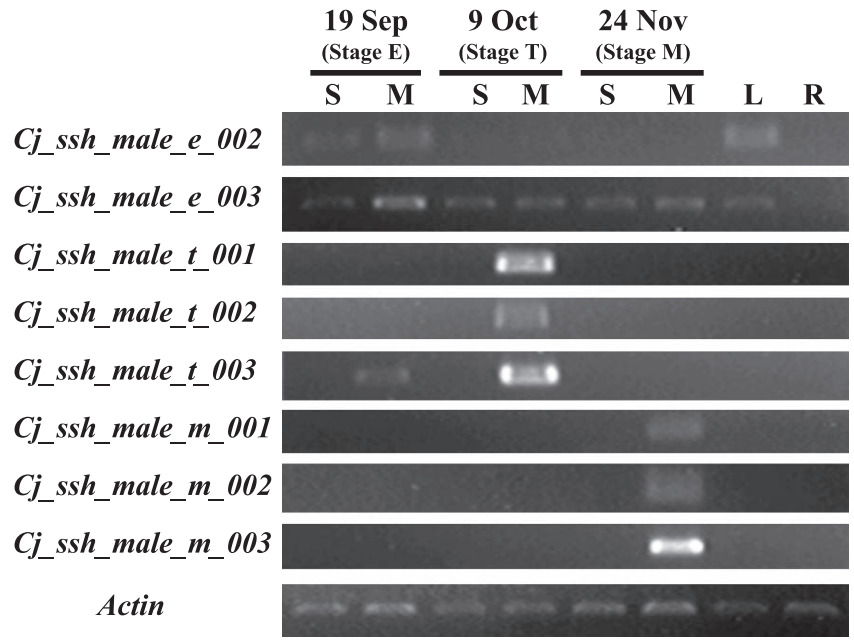

Fig. 4. RT-PCR analysis of genes isolated from subtracted libraries. The template cDNAs were synthesized from shoot (S), male strobilus (M), young shoot (L), and root (R). The samples of shoot and male strobilus were collected on 19 Sep (stage E), 9 Oct (stage T) and 24 Nov (stage M).

veloping gametophytes (Ursin et al. 1991). GA1 promoter activity is highest in rapidly growing tissue; for example, shoot apices, root tips, developing flowers, and seeds (Silverstone et al. 1997). Stage $\mathrm{E}$ is the time when a male strobilus is enlarging. Therefore, we consider that these results show that cell division or protein synthesis was active in stage $\mathrm{E}$.

In stage $T$, a number of genes have sequence similarity with tapetum-specific genes of other species. One of the characteristics of this library is that three kinds of genes of 
Cj ssh male $t$ 001-3 occupied $60 \%$ of all sequences. Two of these genes, $C j \_s s h$ male_t_001 and $C j \_s s h$ male_t_003, have peptide sequence similarity with PrMC1 (Walden et al. 1999) and PrMALE1 (Höfig et al. 2006), respectively, which were isolated from Pinus radiata (Table 2). These genes share similarity with several anther-expressed cDNAs, of which the most well characterized is $A 9$ from Brassica napus and Arabidopsis (Walden et al. 1999). B. napus $A 9$ and Arabidopsis $A 9$ expression is restricted to the tapetum (Paul et al. 1992). Cj_ssh_male_t_017, which has sequence similarity with the Arabidopsis thaliana MS2 gene, was also isolated from this SSH library. The expression of the MS2 gene was observed in the tapetum of wild-type flowers at, and shortly after, the release of microspores from tetrads (Aarts et al. 1997). The tapetal cells have a major role in pollen development by contributing to microspore release, nutrition, pollen wall synthesis, and pollencoat deposition (Bedinger 1992, Goldberg et al. 1993). In the present study, the genes that have sequence similarity with tapetum-specific genes were highly concentrated in the stage T SSH library, which might reflect an increase in tapetum activity in stage $\mathrm{T}$.

A number of genes have sequence similarity with the expressed genes of pollen in stage M. In a study of ESTs from $C$. japonica pollen (Futamura et al. 2006), the existence of the SF18-like gene and CjMP1-related genes in a pollen EST library was reported. Our stage M SSH library also contained these two genes, which occupied $12 \%$ and $5.1 \%$ of all the sequences in this library, respectively (Table 2). Moreover, many sequences had similarity with peroxidase. Flower-specific peroxidase gene, Ghpod, was isolated from cotton (Gossypium hirsutum) (Chen et al. 2009). Ghpod is a member of class III peroxidase genes and is predominantly expressed during pollen development. Chen et al. (2009) presumed that Gphod might play a role in stress response. The data in Fig. 3 show that the stage M SSH library included many genes categorized into response to stress compared with other libraries.

The number of unigenes isolated from each library differed. Genes isolated from the E, T and M SSH libraries numbered 458, 176 and 379, respectively. Because not only the genes for morphogenesis of the male strobilus (e.g., Cj_ssh_male_e_196 (Supplemental Table 1)) but also the genes that contributed to active cell proliferation (e.g., Cj_ssh_male_e_020 (Table 2 and Supplemental Table 1) were included, many kinds of genes might have been isolated from the stage E library. In the stage T library, the variety of genes was greatly decreased. The phenomenon of the decrease in number of expressed genes in the tetrad stage was also observed in other species (Ma et al. 2008). This result might show that pollen development nursed by the tapetum was active in this stage. However, it might also show that other processes cease, such as anther development. In stage $\mathrm{M}$, pollen-specific genes were isolated and the variety of isolated genes increased. In maize, the number of expressed genes increased after meiosis (Ma et al. 2008). These authors considered the anther to be an integrated system, in which activation of anther maturation in the somatic cell layers is contingent on the successful completion of meiosis. Because not only pollen specific-genes but also the anther maturationrelated genes were expressed, the kinds of expressed genes of stage $\mathrm{M}$ might have increased in C. japonica.

This is the first report on spatiotemporal gene profiling of the developmental stage of the male strobilus of C. japonica. Through the analysis of gene expression profiling, we showed that the expressed genes different significantly among developmental stages of the male strobilus. One of our goals is to clarify the broad cascade of gene expression associated with male strobilus development at various developmental stages and in various cell types. Using the results of this study and knowledge of the full-length cDNA library, we will attempt to develop male sterile technology to resolve $C$. japonica pollen-induced pollinosis, which is a serious allergic disease.

\section{Acknowledgements}

We would like to express our gratitude to Yoshie Kawai of the Forest Tree Breeding Center (FTBC) at the Forestry and Forest Products Research Institute (FFPRI) for providing the male strobilus samples, to Dr. Tomonori Hirao of the Forest Bio-research Center at FFPRI for DNA sequencing, and to Eitaro Fukatsu (FTBC, FFPRI) for creating the DDBJ registration files. This research was supported in part by the Agriculture, Forestry and Fisheries Research Council, Japan.

\section{Literature Cited}

Aarts, M.G., R.Hodge, K. Kalantidis, D.Florack, Z.A. Wilson, B.J. Mulligan, W.J.Stiekema, R.Scott and A.Pereira (1997) The Arabidopsis MALE STERILITY 2 protein shares similarity with reductases in elongation/condensation complexes. Plant J. 12: 615623.

Alche,J. D., A.J.Castro, A.Olmedilla, M.C.Fernandez, R.Rodriguez, M.Villalba and M.I.Rodriguez-Garcia (1999) The major olive pollen allergen (Ole e $I$ ) shows both gametophytic and sporophytic expression during anther development, and its synthesis and storage takes place in the RER. J Cell Sci. 112: 2501-2509.

Becker,J.D., L.C.Boavida, J.Carneiro, M.Haury and J.A.Feijó (2003) Transcriptional profiling of Arabidopsis tissues reveals the unique characteristics of the pollen transcriptome. Plant Physiol. 133: 713-725.

Bedinger,P. (1992) The remarkable biology of pollen. Plant Cell. 4: 879-887.

Chen,D., Y.Ding, W.Guo and T.Zhang (2009) Molecular cloning and characterization of a flower-specific class III peroxidase gene in G. Hirsutum. Mol. Biol. Rep. 36: 461-469.

Fukui,M., N.Futamura, Y.Mukai, Y.Wang, A.Nagao and K.Shinohara (2001) Ancestral MADS box genes in Sugi, Cryptomeria japonica D. Don (Taxodiaceae), homologous to the B function genes in angiosperms. Plant Cell Physiol. 42: 566-575.

Futamura, N., T. Ujino-Ihara, M. Nishiguchi, H. Kanamori, K. Yoshimura, M.Sakaguchi and K. Shinohara (2006) Analysis of expressed sequence tags from Cryptomeria japonica pollen reveals 
novel pollen-specific transcripts. Tree Physiol. 26: 1517-1528.

Futamura, N., Y.Totoki, A.Toyoda, T.Igasaki, T.Nanjo, M.Seki, Y. Sakaki, A.Mari, K. Shinozaki and K. Shinohara (2008) Characterization of expressed sequence tags from a full-length enriched cDNA library of Cryptomeria japonica male strobili. BMC Genomics 9: 383.

Goldberg,R.B., T.P.Beals and P.M.Sanders (1993) Anther development: Basic principles and practical applications. Plant Cell. 5: $1217-1229$.

Hashizume,H. (1973) Studies on flower bud formation, flower sex differentiation and their control in conifers. Res. Bull. Tottori Univ. For. 7: 1-139.

Höfig,K.P., R.Möller, L.Donaldson, J.Putterill and C.Walter (2006) Towards male sterility in Pinus radiata - a stilbene synthase approach to genetically engineer nuclear male sterility. Plant Biotechnol. J. 4: 333-343.

Ma,J., D.S.Skibbe, J.Fernandes and V.Walbot (2008) Male reproductive development: gene expression profiling of maize anther and pollen ontogeny. Genome Biol. 9: R181.

Nagao,A., S.Sasaki and R.P.Pharis (1989) Cryptomeria japonica. In: Halevy, A.H. (ed.) CRC Handbook of Flowering, vol. VI, CRC Press; Boca Raton, FL, pp. 247-269.

Paul,W., R.Hodge, S.Smartt, J.Draper and R.Scott (1992) The isolation and characterisation of the tapetum-specific Arabidopsis thaliana A9 gene. Plant Mol. Biol. 19: 611-622.
Shiokawa, T., S. Yamada, N. Futamura, K. Osanai, D. Murasugi, K.Shinohara, S.Kawai, N.Morohoshi, Y.Katayama and S.Kajita (2008) Isolation and functional analysis of the CjNdly gene, a homolog in Cryptomeria japonica of FLORICAULA/LEAFY genes. Tree Physiol. 28: 21-28.

Shukla, A.K., A.K. Shasany, M.M.Gupta and S.P.Khanuja (2006) Transcriptome analysis in Catharanthus roseus leaves and roots for comparative terpenoid indole alkaloid profiles. J. Exp. Bot. 57: 3921-3932.

Silverstone,A.L., C.Chang, E.Krol and T.Sun (1997) Developmental regulation of the gibberellin biosynthetic gene GA 1 in Arabidopsis thaliana. Plant J. 12: 9-19.

Sternberg,M.B. and S.Gepstein (2007) Subtractive hybridization techniques to study cellular senescence. Methods Mol. Biol. 371: 289305.

Taniguchi,T., Y.Ohmiya, M.Kurita, M.Tsubomura and T.Kondo (2008) Regeneration of transgenic Cryptomeria japonica D. Don after Agrobacterium tumefaciens-mediated transformation of embryogenic tissue. Plant Cell Rep. 27: 1461-1466.

Ursin,V.M., J.M.Irvine, W.R.Hiatt and C.K.Shewmaker (1991) Developmental analysis of elongation factor-I $\alpha$ expression in transgenic tobacco. Plant Cell 3: 583-591.

Walden,A.R., C.Walter and R.C.Gardner (1999) Genes expressed in Pinus radiata male cones include homologs to anther-specific and pathogenesis response genes. Plant Physiol. 121: 1103-1116. 